from damage by causing them to revert to 'juvenile' pacemaking mechanisms.

Original article Becker C et al. (2008) Use of antihypertensives and the risk of Parkinson disease. Neurology [doi:10.1212/01.wnl.0000303818.38960.44]

\section{Study finds no link between plasma lipid levels and risk of mild cognitive impairment}

Whether dyslipidemia is associated with an increased risk of dementia is currently a matter of some debate, as studies designed to examine the relationship between plasma lipid levels and cognitive impairment have produced inconsistent results. In a recent study, Reitz et al. found no association between lipid levels and the risk of mild cognitive impairment (MCl) in a cohort of elderly people.

In this prospective, community-based cohort study, the researchers identified 1,772 potential participants by randomly sampling Medicare recipients aged $\geq 65$ years who were resident in northern Manhattan, New York, USA. Only those individuals without prevalent $\mathrm{MCl}$ or dementia at baseline and with available lipid-level data were included in the final analysis $(n=854$; mean age 75.8 years; $69 \%$ female). Patients underwent baseline neurological evaluations and lipid-level measurements during 1992-1994, and followup neurological examinations were conducted at 18 -month intervals.

During 4,189 person-years of follow-up, 153 participants developed amnestic $\mathrm{MCl}$ and 171 participants developed nonamnestic $\mathrm{MCl}$. The mean age of $\mathrm{MCl}$ onset was 80.7 years. Following adjustment for age and sex, higher plasma levels of total cholesterol and LDL cholesterol were associated with a decreased risk of total $\mathrm{MCl}$; however, these associations were weakened when the analyses were adjusted for education, diabetes, heart disease and hypertension. No associations were found between plasma lipid levels and the risks of amnestic or nonamnestic $\mathrm{MCl}$, and treatment with lipid-lowering agents did not affect the risk of $\mathrm{MCl}$.

On the basis of these results from a highly selected cohort of elderly individuals, the authors conclude that dyslipidemia does not increase the risk of $\mathrm{MCl}$ in the elderly.

Original article Reitz C et al. (2008) Plasma lipid levels in the elderly are not associated with the risk of mild cognitive impairment. Dement Geriatr Cogn Disord 25: 232-237

\section{Variants in $A B C B 1$ can predict patient response to antidepressant drugs}

For patients with depression, antidepressant drugs are a principle method of treatment, yet the rate of remission is low. The poor response to antidepressants might to some extent be attributable to inadequate penetration into the CNS, a process that is regulated at the bloodbrain barrier in part by the transporter protein P-glycoprotein (P-gp), a known efflux pump for various drugs. To test this hypothesis, Uhr et al. have investigated the relationship between single-nucleotide polymorphisms (SNPs) in the gene that encodes P-gp (ABCB1) and incidence of remission in patients treated with P-gp-substrate antidepressants.

The study included 443 adult patients who were being treated for depression at a center in Munich, Germany. The authors classified common antidepressants as substrates or nonsubstrates of P-gp by use of an in vivo assay in which these drugs were administered to mice that lacked the homologs of human $A B C B 1$. For patients who were treated with a P-gp-substrate antidepressant (citalopram, paroxetine, amitriptyline or venlafaxine), the presence of any of 11 different SNPs in $A B C B 1$ increased the risk of remission $(P=0.00040$ and $P=0.00016$ for genotypic and allelic analysis, respectively, over all SNPs and all phenotypes). No such association was seen in patients who were treated with a non-P-gp substrate (mirtazapine).

These findings suggest that the combined factors of a patient's $A B C B 1$ genotype and a drug's P-gp-substrate capacity should be taken into account when antidepressants are prescribed.

Original article Uhr M et al. (2008) Polymorphisms in the drug transporter gene $A B C B 1$ predict antidepressant treatment response in depression. Neuron 57: 203-209 